\title{
Erratum to: VAMP4 is required to maintain the ribbon structure of the Golgi apparatus
}

\author{
Akiko Shitara · Toru Shibui • Miki Okayama • \\ Toshiya Arakawa • Itaru Mizoguchi • \\ Yasunori Sakakura • Taishin Takuma
}

Published online: 14 June 2013

(c) Springer Science+Business Media New York 2013

\section{Erratum to: Mol Cell Biochem}

DOI 10.1007/s11010-013-1652-4

In the original version of the article the name of Yasunori Sakakura was displayed as Yasunori Shakakura.

The correct spelling of the name of this author is Yasunori Sakakura.

Everything else in the paper remains correct.

The online version of the original article can be found under doi:10.1007/s11010-013-1652-4.

\footnotetext{
A. Shitara $(\bowtie) \cdot$ T. Arakawa $\cdot$ T. Takuma

Division of Biochemistry, Department of Oral Biology, School of Dentistry, Health Sciences University of Hokkaido, Tobetsu Hokkaido, Ishikari 061-0293, Japan e-mail: shitara@hoku-iryo-u.ac.jp

T. Shibui · Y. Sakakura Division of Anatomy, Department of Oral Growth and Development, School of Dentistry, Health Sciences University of Hokkaido, Tobetsu Hokkaido, Ishikari 061-0293, Japan

M. Okayama · I. Mizoguchi

Division of Orthodontics and Dentofacial Orthopedics, Department of Oral Growth and Development, School of Dentistry, Health Sciences University of Hokkaido, Tobetsu Hokkaido, Ishikari 061-0293, Japan
} 